Alaniz Rodríguez, L. D. (mayo-agosto, 2021). El tratamiento de la infancia en las ciencias sociales: racionalidades e influjos. Revista Virtual Universidad Católica del Norte, (63), 5-38.

\title{
https://www.doi.org/10.35575/rvucn.n63a2
}

\section{El tratamiento de la infancia en las ciencias sociales: racionalidades e influjos}

The treatment of childhood in the Social Sciences: rationalities and influences

\section{Luis Daniel Alaniz Rodríguez}

Magister en Estudios para la Paz y el Desarrollo

Facultad de Ciencias Políticas y Sociales, Universidad Autónoma del Estado de México

Toluca, México

lalanizr001@alumno.uaemex.mx

Orcid: https://orcid.org/0000-0003-1619-3700

Recibido: 02 de julio de 2020

Evaluado: 21 de octubre de 2020

Aprobado: 25 de febrero de 2021

Tipo de artículo: Investigación Científica y Tecnológica

\section{Resumen}

En el presente artículo se realizó un análisis de las racionalidades imperantes, desde el siglo $\mathrm{XX}$, en algunas de las disciplinas y teorías científicas sociales, en su tratamiento de la infancia. Partiendo del debate en torno a la interpretación de Weber, sobre la posibilidad de existencia y coexistencia de distintas racionalidades, se intentó identificar cómo la psicología del desarrollo, la teoría sociológica "tradicional” y la sociología de la infancia han racionalizado sus postulados desde su pretensión científica, y, al mismo tiempo, cómo dichos postulados han influido en la consolidación y desarrollo de políticas e instituciones dedicadas a la socialización de la infancia en América Latina, como la guardería, la escuela y la familia, principalmente. Los resultados de este análisis permiten sugerir que las racionalidades dominantes en las ciencias sociales, así como en las instituciones y sociedades de la región latinoamericana, continúan reproduciendo valores tutelares y adultocéntricos. La posición 
crítica, de corte contrahegemónico, continúa siendo una opción epistemológica mínimamente abordada, y su consolidación se encuentra en ciernes.

Palabras clave: Adultocentrismo; América Latina; Niñez; Racionalidad crítica; Racionalidad tutelar; Socialización.

\begin{abstract}
This article has analyzed the prevailing rationalities since the 20th century in some of the subdisciplines and social scientific theories in their treatment of childhood. Starting from the debate around Weber's interpretation of the possibility of existence and coexistence of different rationalities, an attempt was made to identify how developmental psychology, "traditional" sociological theory and sociology of childhood have rationalized their postulates from their scientific claim and at the same time, how these postulates have influenced in the consolidation and development of policies and institutions dedicated to the socialization of children in Latin America, such as kindergarten, school and family, mainly. The results of this analysis allow us to suggest that the dominant rationalities in the Social Sciences as well as in the institutions and societies of the Latin American region continue to reproduce tutelary and adultcentrist values. The "critical" position, of a counter-hegemonic nature, continues to be an epistemological option that is minimally addressed and its consolidation is in the making.
\end{abstract}

Key Words: Adultcentrism; Latin America; Childhood; Critical rationality; Tutelary rationality; Socialization.

\title{
Introducción
}

Como base de la cientificidad moderna, la racionalidad se ha universalizado para dominar el conocimiento de la humanidad. Como institución colectiva que sienta las bases para la relación entre uno mismo y el otro (Jenks, 2005), la racionalidad ha jugado un rol primordial en el desarrollo de las ciencias sociales y en el tratamiento que estas le han dado a la infancia. En ese sentido, el artículo tiene por objetivo identificar cómo las disciplinas 
científicas han reproducido y producido las doctrinas tutelar y garantista, y, al mismo tiempo, cómo las mismas doctrinas han sido procesadas por las disciplinas científicas en su tratamiento de la infancia. Esto, con el fin de localizar los puntos de quiebre a través de los cuales las racionalidades han ido estableciéndose en la ciencia y en la sociedad, durante el siglo XX y XXI, y también mutando, quizás, hasta encontrar enfoques críticos, como los actuales, con proposiciones claramente diferenciadas a los postulados tutelares.

Para los efectos que se pretenden en este artículo, se hace necesario aclarar lo que se entiende por racionalidad y la manera en que se observa a la misma dentro de las disciplinas abordadas, con el fin de aclarar los presupuestos teóricos aquí empleados. El debate sobre los conceptos de racionalidad y racionalización ha sido nutrido por diversos autores y goza de una amplia tradición; sin embargo, también es conocido que dichos conceptos recobran especial relevancia, en tanto que han sido centrales en la obra de uno de los sociólogos más importantes de la historia, Max Weber. La racionalidad desarrollada por Weber ha sido foco de discusión en la literatura especializada durante las últimas décadas, encontrando también variadas corrientes críticas. A pesar de esto, la racionalidad weberiana puede resultar útil para muchas áreas de investigación, y en este caso, adecuada para el análisis.

Existen múltiples interpretaciones a la obra de Weber en general, así como del concepto de racionalidad, en particular. La interpretación profunda de ambos elementos no corresponde al objetivo de este trabajo; sin embargo, un breve acercamiento al tratamiento conceptual weberiano de racionalidad permite la ubicación en la interpretación que considera que Weber no tenía una noción única ni evolucionista del concepto (Villegas, 2000). Por tanto, se debe, en primer lugar, objetar la interpretación (muy popular, por cierto) que presenta a la racionalidad como fenómeno propio de las sociedades capitalistas y como resultado de un proceso de desarrollo racional que comprende al pensamiento religioso, hasta que alcanza un grado máximo bajo el capitalismo moderno.

La historia no puede sujetarse al significado trascendental del inexorable avance dialéctico de la "Razón" hegeliana o a leyes evolucionistas de cualquier tipo o al eje de una sola esfera institucional, tal como puede ser la Economía. La historia es un laberinto de procesos de racionalización que llegan a institucionalizarse en órdenes legítimos dentro de una sociedad. Algunos de estos procesos convergen, otros chocan, otros más 
se dividen para coincidir en algún momento futuro y algunos llegan a traslaparse surgiendo y luchando con otros procesos en diversas esferas. Por esta razón los distintos procesos no pueden jerarquizarse en un patrón legal de evolución. (Villegas, 2000, p.

Weber (1992) consideró que el capitalismo moderno podría ser visto como la etapa más racional del proceso histórico, pero solo desde la consideración de una racionalidad instrumental. En contraste, podría considerarse al mismo tiempo como una etapa prácticamente irracional, si se le analiza desde la racionalidad sustantiva (Villegas, 2000). Ambas racionalidades serán descritas a continuación, pero esta discusión se retoma especialmente por dos razones que conciernen directamente; estas son que: 1) las racionalidades que se desean identificar dentro de las ciencias sociales en este artículo son propias del capitalismo moderno; y 2) las anteriores afirmaciones ilustran claramente el relativismo y el pluralismo que adoptó Weber en su consideración sobre la racionalidad.

En este sentido, si se abordaran las disciplinas científicas, entendiendo que las mismas se consolidaron paralelamente con el capitalismo industrial, y que a su vez es en el capitalismo industrial y post-industrial ${ }^{1}$, donde se manifiesta la racionalidad "última", se estaría acogiendo la visión reduccionista y evolucionista, donde no quedaría mucho por discutir, más que describir la existencia de una única forma de racionalidad dentro de dichas disciplinas y, por tanto, una manera uniforme de entender la infancia. Por otro lado, la interpretación pluralista permite hacer un análisis donde incluso cabe una suerte de categorización, encontrando rasgos comunes, contrapuestos y/o complementarios entre las distintas racionalidades y las doctrinas tutelar y garantista.

A pesar de que Weber no hace una definición clara y categórica de las distintas racionalidades en su obra, las características de estas pululan en toda su producción (Polisena, 2018); es por ello que gran parte de la discusión y relecturas sobre las mismas han versado sobre la categorización de las racionalidades. Varios autores, incluidos Capera y Ñañez (2017),

\footnotetext{
${ }^{1}$ En referencia con el período histórico que comienza desde siglo XVIII, con la Primera Revolución Industrial británica -etapa en el que las nuevas dinámicas políticas y económicas propiciaron renovados problemas de investigación a través de los cuales sucedió un afianzamiento metodológico que permitió la consolidación de las Ciencias Sociales en el siglo XIX (Kuhn, 1982)- y que va hasta la actualidad "post industrial” (Bell, 1973). Ambos períodos conforman lo que Weber (1992) define como capitalismo moderno.
} 
y Polisena (2018), les abordan desde una división, en dos grandes categorías, estas son: la racionalidad con arreglo a fines, propia de la lógica técnica, el capitalismo moderno y la burocracia (instrumental) y la racionalidad con arreglo a valores, a veces relacionada con el tradicionalismo religioso (sustantiva). Por otro lado, otros han adoptado una visión más amplia de las racionalidades weberianas, incorporando en la discusión otras racionalidades, incluidos Escobar (2020), Fonseca y Prieto (2020). Villegas (2000) ahonda en la obra madura de Weber ${ }^{2}$ y particularmente en Sociología de la religión, para extraer una clasificación basada justamente en una visión relativizada de las racionalidades. Dicha clasificación es útil para comprender las inclinaciones que dan forma a las distintas racionalidades, dentro de las disciplinas de las ciencias sociales durante el siglo XX y lo corrido del siglo XXI, aunque la identificación de alguna de ellas no representa la imposibilidad de hallar otras racionalidades dentro de la misma corriente o disciplina. Así entonces, se encuentran cuatro tipos de racionalidades: la conceptual o teórica, la instrumental, la sustantiva y la formal ${ }^{3}$.

Mediante la racionalidad conceptual/teórica se busca influir y controlar a la realidad de manera consciente, con el fin de otorgarle un orden específico y definido a través de conceptualizaciones precisas y abstractas. En el derecho puede reflejarse en la codificación de proposiciones legales (Villegas, 2000). En este sentido, puede asociarse con términos como "menores en riesgo", "predelincuencia", "socialización” (Jenks, 2005), por ejemplo. Estos conceptos, con cargas semánticas en el fondo muy precisas, pero al mismo tiempo poseedoras de cierta abstracción, fueron influyendo y dando forma a las acciones políticas y sociales hasta convertirse en algo naturalizado, como se verá más adelante.

Por otro lado, la racionalidad instrumental, siendo su auge símbolo mismo del capitalismo moderno (periodo caracterizado por poseer un ethos propio ligado al espíritu productivo y eficiente), se rige por la lógica de medios y fines, donde los medios no son más que instrumentos adecuados para la obtención de los fines concretos (Weber, 1992). En ese escenario, la empresa ocupa un lugar central en la sociedad, y sus valores (rentabilidad,

\footnotetext{
${ }^{2}$ Villegas (2000) se refiere específicamente a los siguientes documentos Sociología de la religión, introducción a La ética económica de las religiones universales, las notas a La ética protestante y el espíritu del capitalismo, La ciencia como vocación y las primeras 45 páginas de Economía y sociedad.

${ }^{3}$ A pesar de tomar como referencia esta clasificación, estás racionalidades no se encuentran exentas de tensiones, disputas y contradicciones. Tampoco son netamente inmóviles ni puras, sino que pueden entrecruzarse y transformarse a través de las prácticas sociales.
} 
inversión, lucro, expansión, acumulación) trastocan las relaciones sociales, especialmente las del sujeto-trabajo-capital. A pesar de estar estrechamente relacionado con formaciones económicas, puede exponerse en el derecho, por ejemplo, a través de un juez con la cualificación necesaria para establecer inocencia o culpabilidad al acusado (Villegas, 2000). Los jueces de menores pueden ser un ejemplo aún más ampliado de esta racionalidad; entre otras, por su potestad extendida, podrían elegir los medios que considerasen adecuados con el fin de "salvar", "curar", "regenerar" al menor en riesgo (Jenks, 2005). La racionalidadformal, por su parte, perfilada con el proceso de industrialización (iniciado en Europa en el siglo XVIII), se constituye como un proceso de despersonalización, basado en reglas abstractas que privilegian la universalización frente al reconocimiento de características personales (Villegas, 2000). El desarrollo científico y tecnológico, la proliferación fabril, el aumento de la población urbana y la consolidación burguesa trajeron consigo nuevas necesidades de eficiencia para afrontar problemáticas cotidianas. La acción, por tanto, se orienta hacia las regulaciones generales, calculando la eficiencia de la misma en relación con la resolución de problemas, otorgando valores que robustecen los aparatos burocráticos ${ }^{4}$ (Villegas, 2000). El nacimiento de la minoridad, así como la psicología del desarrollo, impregnadas de generalizaciones, universalizaciones, presunciones del extracto socioeconómico o presunciones cognitivas (Jenks, 2005), pueden componerse como ejemplos de esta racionalidad.

Con cierta distancia de las anteriores, se encuentra la racionalidad sustantiva. Esta, se define mediante la acción valorativa, es decir, no se ordena de acuerdo con medios y fines ni a generalizaciones universales, sino a postulados de valor (Weber, 1983), y además se ve naturalmente influida por su contexto sociohistórico. Evidentemente, su aplicación depende de las preferencias implícitas o explicitas, conscientes o inconscientes, por ciertos valores éticos (Villegas, 2000). Esta racionalidad retoma la capacidad innata del ser humano para actuar de acuerdo con sus valores, por lo que tiene cierta cercanía con la sociología de la infancia.

La etiqueta de racionalidad con que también se ha identifica aquí lo tutelar y lo crítico se armoniza con la visión pluralista de Weber. Es decir, las bases del paradigma tutelar, al interpretarse desde el enfoque de Weber, se conforman como parte de un sistema amplio a

\footnotetext{
${ }^{4}$ Por su naturaleza, esta racionalidad suele combinarse con la racionalidad instrumental.
} 
través del cual se emplean estrategias puntuales para la consecución de objetivos adultocéntricos ${ }^{5}$ desde distintas esferas, y que se nutre precisamente de postulados racionales, desde lo instrumental, lo formal y lo teórico/conceptual. Sin embargo, la consolidación de lo tutelar no fue un proceso homogéneo, lineal y uniforme; en cada región y país implicó una serie de disputas discursivas y prácticas, especialmente en los escenarios institucionales ${ }^{6}$.

Igualmente ocurre con el posicionamiento crítico que, trascendiendo las bases del paradigma garantista (hacia lo postgarantista), se racionaliza con elementos cercanos a lo sustantivo, para extraer metodológicamente al infante de sus espacios socializadores e independizarle como objeto de estudio. Si bien las categorías weberianas son de carácter social en genérico, encuentran puntos de cruce con el pensamiento científico que construye imaginarios racionalizados de infancia, reproducidos socialmente. Es por ello que la proposición aquí es definir a las corrientes tutelar y crítica como racionales, y emplearles así como grandes bloques analíticos para abordar los distintos tratamientos de la infancia en las ciencias sociales.

El artículo se encuentra dividido en dos secciones principales; en la primera, se abordan las racionalidades imperantes durante el siglo $\mathrm{XX}$, propias de lo tutelar. Aunque no únicamente, estas racionalidades encontraron gran cabida en la psicología y la sociología, y al mismo tiempo, influyeron de manera significativa el pensamiento colectivo, los programas pedagógicos, las instituciones estatales $\mathrm{y}$, por supuesto, las actividades legislativas en América. Después de ello, en una segunda sección, se trata de exponer el nacimiento y desarrollo de una visión influida por las nuevas corrientes sociales cercanas a la teoría crítica y el post-estructuralismo (como la decolonialidad y los feminismos modernos ${ }^{7}$ ) y otros movimientos que aparecen con mucha fuerza en la escena social a finales del siglo XX. Esta

\footnotetext{
${ }^{5}$ Se entiende lo adultocéntrico como las ideas y condiciones autoritarias y de carácter vertical que desconocen y obstruyen el carácter protagónico de los niños y niñas en los procesos de participación y construcción social (Duarte, 2016), tanto de su propia identidad como en la trasformación de su entorno.

${ }^{6}$ El análisis y descripción de los procesos puntuales, diferenciados y particulares en cada región/país requeriría un estudio historiográfico exhaustivo que escapa al objetivo de este trabajo.

${ }^{7}$ El pensamiento decolonial presenta claves para analizar, cuestionar, criticar y trascender la moderna colonialidad del poder, del ser y del saber. Por su parte, los feminismos modernos hacen lo propio frente al patriarcado. Lo que tienen en común es que surgen como posturas críticas frente a sistemas hegemónicos y por demás violentos, es por ello que la racionalidad crítica se influye de ellos para hacer lo propio frente al adultocentrismo.
} 
visión reciente se ha introducido en los estudios sobre infancia y en corrientes de la sociología, principalmente.

\section{Metodología}

La investigación fue de carácter exploratorio y documental, se rigió por lo hermenéutico, lo cualitativo, lo deductivo, y recurrió al análisis de discurso como técnica cualitativa. Se pretendió responder a la pregunta: ¿qué racionalidades son las imperantes en el tratamiento a la infancia en las ciencias sociales y cómo se dan sus influjos? Para ello, el ejercicio se centró en la revisión cualitativa y hermenéutica sobre la posición de la infancia en la literatura académica europea, estadounidense y latinoamericana, particularmente en la revisión teórico-conceptual de la infancia en algunas de las principales corrientes de las ciencias sociales, mediante sus racionalidades, y en el tratamiento social de la misma, a través de las instituciones. En una segunda fase, en concordancia con el objetivo, se abordó un análisis que intentó develar el mutuo influjo que sufrieron disciplinas científicas e instituciones de las sociedades europeas y americanas, particularmente durante la segunda mitad del siglo XX.

Para dicho análisis, se retomaron posturas de la hermenéutica performativa o hermenéutica ontológica política; enfoque epistémico y metodológico que basa sus presupuestos ético-políticos en la filosofía política arendtiana, misma que retoma la teoría sobre el juicio crítico kantiano y la hermenéutica ontológica de Heidegger (Alvarado et al., 2014). Esta, entre otras, rechaza concepciones descontextualizadas, mientras que otorga especial atención al lenguaje como vehículo de inserción del sujeto en el espacio público y como herramienta para recuperar la significación de las actividades, instituciones y experiencias humanas, pensando al discurso no como información únicamente, sino desde su potencial narrativo para representar y recrear los marcos conceptuales históricos y presentes (Alvarado et. al, 2014). Esta hermenéutica resultó especialmente útil en una tercera fase, donde se emprendió una aproximación a las formas de posible mutación que sufrió la racionalidad tutelar hacia un posicionamiento crítico.

El empleo del análisis de discurso como técnica cualitativa (Sayago, 2014) atravesó las tres fases anteriores, en tanto que no se cuantificaron datos recogidos en las fuentes secundarias, sino que funcionaron como punto de partida para encontrar similitudes y diferencias conceptuales 
(Strauss y Corbin, 2002) en las disciplinas estudiadas. A través de una interpretación reflexiva, se analizaron las representaciones discursivas con un ejercicio de etiquetamiento-desagregaciónreagregación, que validó de manera constante las semejanzas y diferencias reconocidas en las unidades de análisis (Sayago, 2014). Ello permitió identificar ciertas generalizaciones, pero también exponer particularizaciones en la manera en que se trata discursivamente a la infancia, con presencias y ausencias conceptuales, que justifican, facilitan u obstaculizan una interpretación de la infancia "ampliada" frente a la tradicional. $\mathrm{Al}$ analizar discursos, entendidos estos como formas de estar en el mundo (Gee, 2005), se da cuenta de posiciones discursivas que no sólo constituyen simples posturas, sino que reflejan entramados relacionales e ideológicos que se manifiestan a través de lo que se dice, pero también en la intencionalidad de lo que se dice y desde el lugar donde se dice (Wetherell y Potter, 1996).

\section{Resultados}

La revisión cualitativa del posicionamiento de la infancia en la literatura académica europea, estadounidense y latinoamericana, particularmente en la psicología del desarrollo, la teoría sociológica y la sociología de la infancia, permite sugerir que, en su tratamiento, la racionalidad tutelar ha sido imperante durante todo el siglo XX en Latinoamérica y ha respondido a intereses de diversos grupos de poder, especialmente el político ${ }^{8}$, haciéndose notar tanto en espacios privados como públicos (Pussetto, 2016). Sólo durante las últimas décadas la racionalidad crítica ha aparecido desde la escena académica; sin embargo, sin la fuerza necesaria para contagiar a variadas disciplinas científicas, menos aún para influir sobre instituciones y sociedad en general. Como derivación de una mezcolanza de las racionalidades instrumental, formal y conceptual/teórica, tanto en las ciencias sociales como en las instituciones y sociedad de la región, se concretó, a partir de esa amalgama, una racionalidad tutelar que se posicionó como la racionalidad científica y socialmente hegemónica. Las disciplinas científicas, al concentrar su investigación en los espacios socializadores más que en la persona, dificultaron el reconocimiento

\footnotetext{
${ }^{8}$ Con referencia a que, en concordancia con los proyectos económicos y de identidad nacional, las instituciones públicas desempeñaron papeles claves en la construcción de una infancia acorde con dichos proyectos, aprovechando su poder e influencia sobre otras instituciones de la sociedad.
} 
del infante como objeto de estudio independiente y como sujeto social con capacidades propias (Osorio, 2016).

Por otro lado, el influjo mutuo y bidireccional entre racionalidades disciplinarias e instituciones de las sociedades latinoamericanas ha sido concreto y decisivo en la creación y desarrollo de políticas dirigidas a la infancia, a la familia, al espacio público, y a todas las instituciones dedicas a la socialización de la infancia en general. En el Cono Sur, durante la época de las dictaduras militares (1954-1990), las políticas dirigidas a las instituciones y espacios socializadores fueron determinantes en la construcción de proyectos nacionales y, por tanto, también más evidentes que en otros países. Las hipótesis científicas de algunas disciplinas sociales se reforzaron desde la laboratización que la guardería y la escuela permitieron (a partir de la década de los años 20 del siglo XX), influyendo directamente en la esfera política (Jenks, 2005). El resultado de ello ha permitido la continuidad de la racionalidad tutelar que, al pasar por instancias estatales, sigue reproduciendo negaciones identitarias hacia aquellas manifestaciones de ser sujetoinfante que no encajen dentro de los prototipos adultocéntricos (Duarte, 2016) y capitalistas (Llobet, 2010).

El surgimiento de la racionalidad crítica, como propuesta conceptual englobadora, es derivación de distintas aportaciones provenientes de la sociología primordialmente, como la mirada poscolonial ajustada a la infancia (Liebel, 2016), la sociología de la infancia (Gaitán, 2006a), entre otras. Estas aportaciones surgen, en primer lugar, conociendo y cuestionando los presupuestos tutelares tan arraigados en las sociedades occidentales, reflejados incluso en las líneas de acción de organismos internacionales (Liebel, 2016). Dichos cuestionamientos - coherentes con las corrientes críticas frente a la cultura hegemónica de la segunda mitad del siglo XX, como el decolonialismo, los feminismos y/o el posestructuralismo-, fueron constituyéndose como fracturas en el pensamiento adultocéntrico (Duarte, 2016), y han abierto la puerta a la posibilidad de mutación hacia una racionalidad crítica. A través de esta última, se trata de entender ahora a la niñez desde su posición de subordinación frente al poder y el mundo adulto, abriendo así espacios para un reconocimiento agencial de la infancia en la vida social (Pavez y Sepúlveda, 2019). 


\section{Discusión. La racionalidad tutelar: la psicología del desarrollo y la influencia de Piaget}

La influencia de Piaget en lo que se constituye como la psicología del desarrollo ha sido determinante, a través, sobre todo, de sus teorías de la inteligencia y el desarrollo infantil. Su visión ha influido globalmente en la atención y práctica pediátrica (Jenks, 2005). Los trabajos de Piaget marcaron un antes y un después en el tratamiento de la infancia occidental, en el periodo entreguerras y la segunda mitad del siglo XX, particularmente. Sus estudios buscaron describir la estructuración del pensamiento y de sus principios racionales a través de la teoría del aprendizaje. Conciliando empirismo y racionalismo, la postura de Piaget (1972b), desde la filosofía, se inserta dentro de la tradición neokantiana, dado que representa un esfuerzo por conciliar empirismo y racionalismo (Jenks, 2005). Piaget (1972b) describió la psicología del desarrollo como el estudio de desarrollo de funciones mentales, en tanto que este desarrollo puede proporcionar una explicación de sus mecanismos en el estado final. Es decir, la psicología del desarrollo consiste en recurrir a la psicología infantil para hallar soluciones a los problemas psicológicos generales.

Primordialmente, Piaget (1972b) sugirió que las competencias cognitivas del infante siguen una secuencia universal. Dicha secuencia se mide sobre la capacidad progresiva de pensar el mundo de acuerdo con los valores, principios y conceptos de la filosofía occidental, particularmente las categorías kantianas de espacio, tiempo y causalidad (Jenks, 2005). Por desarrollo se entiende un sistema particular de racionalidad científica, de valores eurocentristas, que se presenta como algo universal y, además, natural (Jenks, 2005). Las etapas de dicho desarrollo son ineludibles y concretas, inician con la inteligencia sensorialmotora, continúan con el pensamiento preconceptual o preoperacional, las operaciones concretas y, finalmente, las operaciones formales (Piaget, 1972a).

La racionalidad científica es entendida por Piaget como el proceso lógico-deductivo, la matematización y las operaciones cognitivas (Jenks, 2005, p. 24). Esta epistemología genética imposibilita al infante el diferenciarse dentro del reino de la racionalidad universal, propia de una racionalidad formal, en tanto que aplana cualquier curva anormal en el desarrollo. "Los niños reales ubicados históricamente están sujetos a la violencia de un modo contemporáneo 
de racionalidad científica que se reproduce, a expensas de su diferencia" (Jenks, 2005, p. 23). Estas diferencias son reprimidas a través de la estandarización del desarrollo.

La psicología del desarrollo debe su posibilidad de avance gracias a la guardería y a la clínica. A través de ellas -especialmente desde la década de los años veinte en adelante-, se permitió la observación y la experimentación controlada de una gran cantidad de infantes de distintas edades. Ello conllevó a una estandarización y construcción de normas de desarrollo basadas en habilidades promedio, según las edades y determinadas actividades (Rose, 1989, como se citó en Jenks, 2005). Por tanto, toda esta laboratización permitió, desde principios científicos y apoyándose en instituciones, la constitución de imágenes de normalidad y anormalidad en cada etapa etaria de la infancia. Dicha constitución resultó de los procesos de disputas de saberes dentro de los campos profesionales, como la pedagogía, la psicología y la pediatría; para el caso argentino, ello sucedió en la primera mitad del siglo XX (Lionetti, 2018). Esta relación recursiva entre ciencia (psicología del desarrollo) e instituciones en la interpretación de la infancia se fortaleció gracias, entre otras, a la racionalidad formal, que desde el discurso incidió en la educación familiar y escolar.

El pensamiento de Piaget y sus postulados normativos dentro la psicología del desarrollo han tenido una muy significativa influencia, tanto en las ciencias sociales como en las instituciones dedicadas a la socialización y al aprendizaje en Occidente. Esto permitió cierto monopolio sobre la visión general de la infancia en la conceptualización cotidiana del mismo (Jenks, 2005), siendo el enfoque dominante durante el siglo XX en el mundo occidental, al mismo tiempo que reforzó el carácter científico de la psicología en el área infantil. La racionalidad formal jugó en este proceso un papel determinante.

La constitución de la psicología como disciplina científica tiene una estrecha relación con el estudio de la niñez, sobre todo en su afán de medida y clasificación de la misma. Las principales tesis psicológicas se han basado especialmente sobre la otra niñez o menores, es decir, sobre los sujetos infantiles sumidos en algún tipo de vulnerabilidad social (Llobet, 2010), mayoritariamente socioeconómica. Se encuentra en ello mucha cercanía con la minoridad de finales de siglo XIX, asociada al riesgo, a la predelincuencia, a las conductas "asociales" e "irracionales"; todos ellos conceptos tutelares y propios de la racionalidad conceptual/teórica. 
En el centro de la categoría, parece posible encontrar invariantes estructurales, una suerte de 'esencia' de la niñez en tanto momento del desarrollo, que no se ve afectada por las transformaciones histórico-sociales. La segunda consecuencia, derivada de la primera, es que las transformaciones subjetivas determinadas socialmente son traducidas en problemas psicopatológicos (internos al campo psicológico como variedades anormales del objeto) o problemas sociales (externos al campo de conocimiento y, por lo tanto, ajenos). (Llobet, 2010, p. 9)

La concentración en los patológico incentivó la inclusión de conceptos y criterios clínicos y psicopatológicos como marcos para el desarrollo de políticas sociales dirigidas a la infancia. Es justamente por ello que las políticas públicas se han regido por una perspectiva y preocupación psicopatológica, derivada de lo normal y anormal, mientras que la tensión entre autonomía y dependencia, también problema crucial del campo psicológico no fue atendido plenamente, relegándola a un sitio marginal (Llobet, 2010). Desde la racionalidad conceptual/teórica, el universo conceptual de la psicología del desarrollo estableció necesidad, normalidad y deseabilidad en el desarrollo del sujeto (Jenks, 2009) y la relación niños-adultos. Estos conceptos permearon el desarrollo de políticas públicas dirigidas a la infancia en América Latina, así como el desarrollo de actividades científicas infantiles y juveniles en esta región (Lozano et al., 2016).

Una primera ruptura en la relación niños-adultos ha sucedido, según Llobet (2010), durante los años sesenta del siglo XX, derivado del clima político y cultural sucedido en dicha década, donde el psicoanálisis y la pedagogía con influjo de la psicología fueron sustituyendo a la moral. A través de esta renovación, el niño dejaría de ser tratado como una tabula rasa donde su contenido mental solo era incorporado a través del desarrollo, y pasaría a preguntarse con mayor formalidad sobre sus motivaciones e intenciones propias; es decir, el niño era visto antes como una superficie opaca sin pretensiones, y durante esta etapa comenzó a ser más como una suerte de incógnita para el mundo adulto. Esta visión, a pesar de representar una ruptura o giro en la interpretación del infante, no dejó de guiarse por una visión adultocéntrica propia de lo tutelar.

Esta renovación fue base para un giro intimista ligado a los postulados del psicoanálisis, otorgando a los padres un lugar más protagónico sobre la salud de sus hijos (conservando los 
preceptos de normalidad y anomalidad), responsabilidad que se había desplazado a las instituciones desde fines del siglo XIX y principios del siglo XX, con base en la higiene y la concepción de la infancia como bien público. Por tanto, en este renovado modelo de familiaridad el niño regresa con importancia al ámbito de lo privado, inyectando al mismo tiempo una incertidumbre sobre las nuevas formas y estrategias pedagógicas que padres y madres necesitaban adoptar para garantizar un cuidado adecuado de los infantes (Llobet, 2010). En este sentido, puede verse como, a pesar del nacimiento de nuevas interrogantes sobre la infancia, los postulados de la psicología, la pedagogía y, más recientemente, el psicoanálisis tuvieron influjo directo sobre la educación formal e incluso en los modelos de familiaridad en la segunda mitad del siglo XX, coincidiendo con la etapa madura de la obra de Piaget.

Por otro lado, se popularizaba una nueva conceptualización. En los discursos políticos, marcadamente en América Latina, la denominación de menores comenzó a vincularse con mayor frecuencia con la familia, y paulatinamente se vio reemplazada por la expresión menores y familia, que empezó a usarse recurrentemente (Llobet 2010). La necesidad de reintegración familiar de los menores también ocupó un lugar importante en el debate sobre la infancia en aquella época. Como evidencia del influjo del giro intimista en la sociedad, así como de la psicología y pedagogía, surgieron en Argentina $^{9}$ el Estudio del Niño y el Adolescente Institucionalizado, en 1967, y el Congreso del Menor Abandonado, en 1969; se publica la Política Nacional del Menor y la Familia en 1973, y más herramientas de información que comienzan a indagar sobre distintas formas de abandono, maltrato hacia los infantes, e idealizan el comportamiento del infante y la familia en la sociedad de la segunda mitad del siglo XX.

En el Cono Sur, en la época de las dictaduras militares, se inauguran estrategias de tratamiento, como registros generales de menores, orientación familiar, becas para internamiento en instituciones privadas, pequeños hogares y becas de estudio para ingreso a instituciones militares (Dubaniewicz, 1997). De estas iniciativas de naturaleza preventiva y tutelar, nacen categorizaciones como niños con problemas graves o niños sin problemas, que

\footnotetext{
${ }^{9}$ Cabe señalar que en el caso argentino la minoridad se fue consolidando paralelamente con el aparato institucional estatal desde fines del siglo XIX, consolidaciones ambas enlazadas a la reconfiguración económica (incluido el trabajo infantil en la calle) y la explosión demográfica urbana de la época (Aversa, 2016).
} 
fueron determinantes para el nacimiento de los regímenes técnico-políticos de familias sustitutas (Llobet, 2010), por ejemplo. En Brasil, los primeros en difundir el ideario de infancia según los valores burgueses fueron los médicos, durante las primeras décadas del siglo XX (Fávero, 2018). A través del Código de Menores de 1927, se definió en Brasil el campo jurídico que daría bases para la creación de instituciones dedicadas al menor abandonado, al menor trabajador y al menor delincuente (Fávero, 2018). Durante las décadas siguientes, proliferaron encuentros políticos, sociales y científicos para debatir sobre las políticas públicas dirigidas a la infancia.

La ola de institucionalización influida por las disciplinas científicas y sobre todo la psicología, como forma de control social y a pesar del giro intimista, continuó con la tradición tutelar de racionalidad formal que no dirigía su control a través del castigo físico, sino con un ejercicio de poder encaminado a negar identidades (Llobet, 2010), donde el encuentro y cultura públicos pasaron de ser lugares de socialización a ser lugares de terror, y donde la población en riesgo se planteó como una amenaza al plan universal del proyecto totalitario de lo que suponía ser infante y familia, modelo en los regímenes militares de la época (Llobet, 2010). Desde la racionalidad tutelar, los espacios de socialización natural del niño no deben ir más allá de la escuela y la familia, por lo que se sancionan los encuentros infantiles fuera de dichos ámbitos. En este sentido, la asociación siempre sería controlada y vigilada por adultos racionales. Esto reforzó el carácter disciplinador de la escuela (Bourdieu y Passeron, 2008), reproduciendo un pensamiento único y universal que mantuvo una lógica de infantilización (Ballatán y Campanini, 2008).

El reconocimiento y tratamiento de la infancia moderna, en el caso argentino, así como en el grueso de la región, se construyó mediante diferenciaciones jurídicas, mecanismos políticos, culturales y científicos, intervenciones normalizadoras y estigmatizadoras que surgieron desde fines del siglo XIX y todo el siglo XX en el escenario estatal (Zapiola, 2018). En la construcción de la historia de la infancia se ha privilegiado esta mirada que da cuenta de las relaciones Estadoinfancia y de la incidencia directa de las leyes, las instituciones y de agentes estatales en la configuración de la experiencia infantil, especialmente de la infancia popular (Zapiola, 2018). Todo ello fue resultado de tensiones, disputas y contradicciones constantes dentro de los campos de los 
saberes $^{10}$ y espacios institucionales, siendo esto parte de largos y complejos procesos socioculturales que, generalmente, ubicaron al infante como objeto de discursos y no como agente histórico (Zapiola, 2018).

Así, entonces, ciencia e instituciones experimentaron una relación racional recursiva, en tanto que, los conceptos científicos (racionalidad conceptual/teórica) influyeron en las instituciones de socialización y sus aparatos burocráticos, universalizando, generalizando y naturalizando al infante (racionalidad formal). Finalmente, dichos procesos institucionales se consolidaron como ambientes propicios para la investigación científica. Se observó igualmente que los planteamientos más importantes de la psicología del desarrollo provienen, intencional o ingenuamente, de un interés por ubicar al infante como ejemplo para demostrar y dar continuidad a fundamentos particulares de ciertas versiones de humanidad, racionalidad, orden, acción, lenguaje (Jenks, 2005), con la evidente pretensión de universalizarles y de que sean instrumentalmente útiles para tales versiones, bajo la lógica de la racionalidad instrumental.

Recapitulando, el desarrollo psicológico e intelectual es lineal, progresivo, regular, acumulativo y de complejidad gradual, y tiene siempre como destino el estadio de la racionalidad científica lógico-matemática, basado en valores filosóficos occidentales. Por tanto, desconoce diferencias, irregularidades, regresiones, disparidades, posibilidad de coexistencia entre múltiples racionalidades, conocimiento narrativo, entre otros muchos elementos que no encajan en la lógica cientificista y la racionalidad formal. Lo que sí es una constante, es el riesgo del infante derivado de su irracionalidad, de ahí la necesidad de tutelarle. Por tanto, la doctrina tutelar se nutre íntimamente de las racionalidades formal, conceptual/teórica e instrumental.

Por otro lado, el giro intimista no demostró ser una transición que dejó atrás la racionalidad tutelar, sino sencillamente representó el desplazamiento de la primera responsabilidad sobre la infancia de ciertas instituciones a otras, en este caso, del Estado hacia la familia. Todo ello sin dejar atrás la visión que defiende el tutelaje como forma de contener vulnerabilidades, potenciales comportamientos delictivos e insurgentes, autonomía,

\footnotetext{
${ }^{10}$ Siguiendo a Zapiola (2018), el campo histórico de la infancia se ha producido mayoritariamente como resultado del proceso dialógico entre la antropología, la sociología, la psicología, el derecho, la educación y los estudios culturales y literarios.
} 
patologías psicológicas y expresiones de agencia. Es decir, el giro intimista reprodujo los criterios de necesidad, deseabilidad y normalidad en el infante, pero adaptados a un discurso político y a un proyecto nacional asiduo de formación identitaria, sobre todo durante las dictaduras, que permitiera la reproducción ininterrumpida del orden social en construcción para la época.

\section{La infancia en la teoría sociológica}

La teoría sociológica tradicional ${ }^{11}$, por su natural inclinación hacia el determinismo y las explicaciones generales (Bajoit, 2008), ha servido, voluntaria o involuntariamente, a naturalizar al infante en cada época, reduciendo o deshabilitando una comprensión más profunda de los niños dentro de su contexto sociohistórico particular (Jenks, 1989). Parece que la infancia ha estado sumida y más bien olvidada, al igual que la interpretación profunda del sujeto, en la preocupación central de la teoría clásica, con claros rasgos de una racionalidad conceptual/teórica. La infancia, entendida como el espacio social de la vida los niños y niñas, en la teoría sociológica tradicional, ha estado recluida dentro de otros subcampos sociológicos como familia, socialización y educación, primordialmente (Rodríguez, 2003).

A pesar de ello, en el marco de varias teorías sociológicas se han abordado cuestiones relativas al desarrollo de la identidad y el desarrollo infantil desde el interaccionismo (Mead, 1982), desde la teoría de las generaciones (Mannheim, 1928/2012), desde el funcionalismo (Parsons, 1951) o la acción educativa (Durkheim, 1922/1956). Sin embargo, en la mayoría, estas aproximaciones, con mayor o menor intensidad, la infancia solo sirvió como un instrumento en el afán de explicar otros conceptos y teorías (Rodríguez, 2003) relacionados con la perspectiva de la reproducción del orden social (Neustader, 1989).

Con una voluntad dependiente a la supraindividualidad estructural que es restrictiva y determinista, "la tradición de la sociología tiene poca pretensión de proporcionar una teoría sólida del individuo y esto tiene implicaciones para nuestra comprensión del niño" (Jenks, 1989, p. 94). Además de esta primera restricción, la teoría sociológica determina causalidades estructurales a

\footnotetext{
${ }^{11}$ Con referencia a las teorías con marcada inclinación hacia las explicaciones de corte estructuralista y funcionalista, concentradas en las instituciones como la familia, escuela, trabajo, religión, política, entre otras, y de gran influencia particularmente antes de la crisis de los años setenta (Bajoit, 2008).
} 
partir de sistemas construidos con base en conductas, normas y costumbres de los adultos racionales y, además, típicos. Si el sujeto adulto sufre aquí de cierta marginación, en el sentido del estudio subjetivista como actor-individuo-sujeto (Bajoit, 2008), el infante se encuentra totalmente abandonado, por así decirlo. La niñez, entonces, entra sencillamente en el terreno de lo patológico o insuficiente, en relación con el modelo prototípico del agente/actor social (Jenks, 1989).

Las teorías que empiezan a preocuparse por el infante, más adelante, definirán al infante con base, únicamente, en la socialización ${ }^{12}$ (como proceso de inducción al mundo social), mayoritariamente familiar y escolar. La socialización ha sido determinante para la adopción de patrones del sistema adecuados para la transformación en un hombre adulto como sujeto acondicionado por la estructura funcional. Mediante la socialización se obliga a la interiorización del sistema normativo, desde el rígido determinismo cultural (Rodríguez, 2003).

Durkheim ha sido de los primeros sociólogos con interés por estudiar a la infancia. En concordancia con su época, concentró su estudio en las instituciones y prácticas educativas más que en la niñez como objeto en sí misma (Gaitán, 2013). Durkheim (1922/1956) identificó a la acción educativa como un proceso mediante el cual se supera la naturaleza inacabada, borrosa, difusa y pasiva del infante, acercándole así hacia la rectitud moral propia de la adultez. En este sentido, la educación representa una suerte de enlace entre el individuo y su sociedad; enlace dirigido a condicionar una reproducción apropiada del orden social. La acción educativa construye al individuo, en tanto que a través de ella se interiorizan la cultura y las normas que son necesarias para la socialización, es decir, su integración a la sociedad (Gaitán, 2013). Este proceso no es complaciente, es un ejercicio de presión de la sociedad misma sobre sus nuevos miembros. Esta educación debe imprimir cierto control sobre los impulsos y deseos del infante, es por ello que dicha tarea socializadora debe pertenecer a la sociedad, a través del Estado, dado que la autoridad debe ser exterior al ambiente familiar, mismo que es propicio a la indulgencia y a la calidez.

Por su parte, Parsons (1951) entiende que los patrones de orientación y valor más estables y duraderos se establecen durante la infancia, pero incluso es posible ver que la socialización de

\footnotetext{
12 "Socialización" puede significar dos cosas muy distintas; cuando se confunden, el resultado es una visión demasiado socializada del hombre. Por un lado, la socialización significa la "transmisión de la cultura", la cultura particular de la sociedad a la que un individuo ingresa al nacer; por otro lado, el término significaba el "proceso de convertirse en humano" de adquirir atributos humanos únicos de la interacción con los demás. Desde la teoría social clásica, parece que socialización fue tomada en ambos sentidos (Wrong, 1961).
} 
cierta manera se acercaba mucho con el concepto de desarrollo infantil: "El término socialización en su uso actual en la literatura se refiere principalmente al proceso de desarrollo infantill" (Parsons, 1951, p. 13). El estructural funcionalismo parsoniano se constituye primordialmente de dos metáforas, lo organicista y el sistema. El primero se refiere a lo inespecífico, lo vivo y al contenido, mientras que el segundo, a lo explícito, lo inanimado y a la forma. "Es como si las sociedades fueran concebidas como organismos vivos, pero en todas partes se están convirtiendo en máquinas" (Jenks, 2005, p. 14). En este planteamiento, la sociedad se convierte en la unidad total que aplica orden y que se autorregula mediante normas y reglas de conducta de forma cada vez más mecánica, infiltrando su existencia supraindividual en las voluntades individuales y siendo la originaria de toda causalidad. Las diferencias, entonces, se funden en la colectividad ordenada y funcional, donde el consenso social somete las conciencias críticas individuales, instalando una racionalidad formal.

Bajo esta lógica, el niño debe ser incluido en los dictados del sistema social, a través de la socialización y el tutelaje, en tanto que su estado natural o no socializado, le presenta como un descarriado, disparejo, alguien no funcional, casi humanamente inacabado, que representa un riesgo potencial frente a las normas sociales y la identidad de la sociedad. Por tanto, el infante debe transitar paulatinamente hacia el mundo adulto (maduro y competente) para convertirse completamente en humano (Jenks, 2005, p. 19).

Las racionalidades, diseñadas y manipuladas en ocasiones, sirven a la exclusión de ciertos grupos, en este caso la racionalidad adulta que ha influido en la teoría pedagógica y la planificación curricular, sobre todo a través de la socialización. Esto puede evidenciarse, por ejemplo, en el modelo educativo (liberal y racional) defendido desde los años setenta por Hirst y Peters (como se citó en Jenks, 2005), con base en máximas universales “necesarias", para desarrollar conocimientos y que al final presentaban justificaciones para los órdenes sociales preexistentes y actuales. En este aspecto, la socialización, en sentido parsoniano, se formalizó mediante muchos programas educativos, en concordancia con una racionalidad formal, que en palabras de Jenks (2005) se destacó como "un proceso violento y doloroso en el sentido altamente político de que todas las personas están obligadas a convertirse en algunas categorías de ser en lugar de otras” (p. 20).

Por otro lado, desde el interaccionismo simbólico, Mead (1982) también abordó el proceso de socialización, pero con una marcada inclinación hacia el carácter conductual del infante. Desde 
dicha perspectiva, los infantes ya pueden verse como manipuladores e interpretadores de la colección de normas y valores culturales del grupo social en el que se desarrolla su aprendizaje (Rodríguez, 2007). Mediante su participación en el proceso de socialización, el infante participa de manera activa en la formación de su propia conciencia e identidad, a través de actividades como el play \& game (Gaitán, 2013), en un proceso de auto interacción simbólica. El núcleo de la socialización es para Mead (1982) la interacción, en tanto que el desarrollo biológico por sí mismo es infructuoso, sino se activa la experiencia de la interacción. Aunque la interacción sigue siendo lo primordial para Mead (1982), el infante comienza a cobrar una relevancia distinta, una relevancia en sí mismo. Su pasividad comienza a sufrir aquí una mutación. A pesar de que su planteamiento se aleja considerablemente del funcionalismo o el desarrollo de Piaget, Mead (1982) no descarta a la socialización como herramienta de control social y de "ajuste" hacia la incorporación del orden prevalente.

\section{La racionalidad crítica: la sociología de la infancia}

El planteamiento de Mead (1982) impulsó nuevos enfoques que alimentaron la sociología de la infancia, definida esta por Gaitán (2006a) como una subdisciplina sociológica. Esto, evidenciando la evolución histórica por la que han transitado los estudios de la infancia en las ciencias sociales desde principios del siglo XX, con Benedict y Mead. Gaitán encontró que tradicionalmente la sociología no consideró a la infancia como objeto formal de estudio, sino más bien se le ubicó en un rol instrumental, siendo la temática central el análisis del sistema social o las instituciones sociales concernientes a la socialización de la infancia. Esta visión:

Cambia cuando se acepta que la infancia constituye una parte permanente de la estructura social que interactúa con otras partes de esa estructura; y que los niños se encuentran afectados por las mismas fuerzas políticas y económicas que los adultos y están sujetos, igual que estos, a los avatares del cambio social. (Gaitán, 2006a, p. 10)

Esta última forma de ver la infancia es la que comienza a ser más abordada con autores como Jenks (1982), con el fin de demostrar que no existía un consenso definitivo de la infancia en la teoría social y, además, que la infancia ocupaba un lugar intencionado que servía a perpetuar 
estereotipos de hombre, orden, lenguaje y acción. La idea de infancia como construcción social cobra fuerza y se desarrollan investigaciones que relacionan a los niños con el sistema económico y la política social. Este impulso fue novedoso en tanto que se empieza a estudiar la infancia desde lo sociológico, como objeto de estudio en sí mismo, y no solo desde la psicología o la educación; se habla de los años ochenta del siglo XX, en adelante. Durante esta época se crearon y desarrollaron proyectos como las publicaciones de la Sociological Studies of Child Development en 1986, el Programa de la Infancia del Centro Europeo para el Bienestar Social en 1987, el Seminario Europeo sobre Investigación y Políticas de Infancia en 1991, la revista Childhood: A Global Journal of Child Research en 1993 (Gaitán, 2006a), observando así cierto influjo académico sobre las instituciones.

Gaitán (2006a) ubicó tres enfoques predominantes en los estudios de la infancia. El primero, sociología de los niños, que tiene como premisas ver a los niños y niñas como agentes que participan en la construcción de conocimiento, como actores sociales participativos y propositivos; y propugna porque las investigaciones se centren en ellos y en la intersección de sus relaciones con otros niños y con los adultos. El segundo, la sociología deconstructiva de la infancia, entiende que las nociones sobre infancia han sido construcciones discursivas que se comunican de diversas maneras en la vida social. La principal intención de este enfoque es deconstruir el poder discursivo dominante y ofrecer nociones de infancia desde espectros culturales más amplios, donde niños y niñas también son considerados como agentes que modelan estructuras y procesos sociales en sus entornos. Por último, la sociología estructural de la infancia define a la infancia como elemento permanente de la estructura social y como una estructura por sí misma, en una misma categoría que la clase o el género. Su intención es relacionar los aspectos de la vida de los niños y sus efectos con un nivel macrosocial.

Otra clasificación a nivel de planteamientos, son el estructural, el constructivista y el relacional. Estos, se ilustran brevemente en la expresión de Gaitán (2006a):

Mientras el enfoque estructural hace hincapié en demostrar la parte más abstracta del binomio persona-sociedad (aquí más bien sociedad-persona), el enfoque relacional insiste (de forma propositiva) en la necesidad de reconocer un papel para los niños, moviéndose el enfoque construccionista dentro de un cierto equilibrio en su intento de definición de ambos conceptos. (p. 16) 
El reconocimiento de la infancia como concepto en singular, puede relacionarse con un planteamiento estructural, dado que se reconoce en un corte transversal y paralelo a otros estadios sociales, como la adultez y la vejez, que son al tiempo atravesados por sistemas verticales como la clase, el poder, el género, la raza y otras categorías jerárquicas que dan cuerpo a las estructuras (Gaitán, 2006b). Un ejemplo de ello es el empleo de la palabra agentes $^{13}$, más recurrente en el planteamiento del enfoque relacional, mientras que en el estructural es actor social y en el construccionista "agency". Una de las ventajas del planteamiento de enfoque estructural es que pretende vencer la invisibilidad social de la infancia como generación, reconociendo al mismo tiempo a la adultez y la vejez como pares, algo que se pierde en los otros dos enfoques, más concentrados en explicar la relación niñoadulto de forma más bien microsocial.

Nuevas tendencias en las aproximaciones sociológicas a los estudios sobre infancia se han desarrollado durante los últimos años, retomando postulados de corte garantista y postgarantista, donde los derechos de la infancia tienen amplias implicaciones. En estas nuevas tendencias se comienza a ver a la infancia como una realidad social permanente de la estructura social y se trasciende el enfoque de socialización, que pasa más a ser un instrumento de análisis (Gaitán, 2006a), donde ahora lo central es el infante como sujeto y su consideración como agente/actor social, en concordancia con una racionalidad sustantiva que revalora a la infancia desde su humanidad y su contexto sociohistórico. Este impulso crítico, de corte contrahegemónico, como el de la sociología de la infancia, adopta la preocupación por integrar cuerpos teóricos con fines argumentativos (Osorio, 2016) que trasciendan la visión hegemónica ${ }^{14}$ sobre la infancia. Es decir, lo contrahegemónico se presenta aquí como las grietas sociales e intelectuales que cuestionan los mecanismos de subordinación y dominación que constituyen los procesos tutelares, resistiendo, limitando, alterando y desafiando las dinámicas hegemónicas que construyen nuestros sentidos de la realidad (Williams, 1997).

\footnotetext{
${ }^{13}$ Por ejemplo, en el desarrollo conceptual de la agencia, dentro de la teoría de la estructuración, Giddens usa indistintamente agente y actor (Giddens, 1984/1995, p. 24).

${ }^{14}$ Se entiende como los procesos de dominación y dirigencia intrínseca a la lucha de clases y las instituciones (Gramsci, 1999), representando aquí la manera dominante de pensar la infancia. Esta hegemonía debe ser renovada, recreada, modificada y defendida de manera continua (Williams, 1997).
} 


\section{Poscolonialismo y otras miradas críticas}

En concordancia con la irrupción contrahegemónica, Plesnicar (2017) ha dado cuenta de cómo Liebel ha encontrado que, desde los años setenta y ochenta del siglo XX, principalmente con los escritos de Mendel (1971) y Gstettner (1981), se inició una reflexión que relacionaba el concepto de colonización con infancia; relacionamiento muy valioso, sobre todo, dado que en aquella época no existían aún teorías decoloniales latinoamericanas ${ }^{15}$. De esta manera, retoma el paralelismo entre el discurso occidental y eurocentrista que otorga derecho moral para colonizar, civilizar, educar a pueblos originarios, y cómo este mismo argumento se usa y ha usado hacia la infancia. Valiéndose de algunas de las teorías decoloniales más importantes, como la violencia epistémica de Boaventura de Sousa, la alteridad de Said, el posoccidentalismo de Mignolo, entre otros, Plesnicar (2017) revalidó (como en una segunda ola, por así decirlo) que la colonización de la infancia sigue vigente, influyendo y materializándose a través de las políticas de la niñez con un carácter universalista (OMS, UNICEF), menospreciando formas de vida distintas a la europea. Este paternalismo intrínsecamente tutelar, según Plesnicar (2017), se encuentra estrechamente ligado a la historia colonial.

En este sentido, las constelaciones de poder neocoloniales influyen en la vida de los niños y niñas y en las construcciones sobre la infancia. Por tanto, la necesidad de las perspectivas poscoloniales se justifica porque:

El imperio del Norte sobre el Sur está entrelazado de manera inseparable con los patrones de infancia predominantes en el Norte. Es con estos patrones que se mide y evalúa las infancias en el Sur global, calificándolas como deficientes, lo cual es problemático. 2) El predominio normativo de la(s) infancia(s) del Norte trae consigo una sobreproducción (y sobrevaloración) de conocimiento que a través de la pretensión de conocer la verdad queda ciego frente al eurocentrismo que implícitamente transporta; los enfoques poscoloniales podrían socavar y revertir este proceso. 3) Siempre y cuando tome en cuenta estas dos limitaciones, el análisis

\footnotetext{
${ }^{15}$ Las teorías decoloniales surgen con fuerza en la escena social latinoamericana en los años finales del siglo XX y principios del siglo XXI.
} 
de la capacidad de acción o actoría (agency en inglés) de niñas y niños puede superar la falta de imaginación en cuanto a sus implicaciones sociales, políticas y éticas y llegar a generar formas de actoría que vayan mucho más allá de lo que abarcan los patrones de infancia comunes del Norte. (Nieuwenhuys, 2013, como se citó en Liebel, 2016, p. 254)

Los seres colonizados equivalen a infantes que deben desarrollarse, introduciendo una separación jerárquica entre adultos y niños, ejerciendo así una suerte de violencia epistémica que coarta las capacidades y posibilidades del actuar humano, prolongando el poder colonial. Esta relación exige a los infantes a verse a sí mismos con la mirada de aquellos que despliegan poder sobre ellos (Cannella \& Viruru, 2004). La subordinación se refuerza, ya que la misma construcción científica ha objetivizado este pensamiento binario. Este patrón se pretende universal desde las sociedades burguesas europeas, e influye de manera directa en las políticas de gobierno, en las ONG y en toda la maquinaria de cooperación al desarrollo en América Latina.

Los patrones indígenas o rurales en Latinoamérica se contraponen al patrón europeo burgués, dado que el trabajo, la co-responsabilidad y la interdependencia se generan desde edades tempranas y, según Punch (2001), dan origen a sentimientos de orgullo e identidad que facilitan la inserción del niño y niña en la vida comunitaria. En contraposición con la psicología del desarrollo, Punch (2001) expone que la recreación, el trabajo y el estudio no son incompatibles, sino complementarios en el desarrollo social comunitario, donde el trabajo no representa un martirio, sino una satisfacción, en una relación caracterizada por la responsabilidad compartida que fortalece sentido de comunidad, aceptando pluralismo y diversidad, bajo la lógica de la racionalidad sustantiva.

El trabajo infantil y adolescente, en el caso de algunas culturas indígenas, es una virtud en el proceso de socialización y produce una alta autoestima en los niños y niñas (García, 2010). Es decir, incluyen aquí otros espacios de socialización, como el trabajo, donde no necesariamente la interacción con el adulto es una relación de tutelaje, sino de coresponsabilidad. Sin necesidad de apologizar el trabajo infantil, dichos espacios demuestran la existencia de estructuras de infancia netamente distintas al patrón eurocentrista (Liebel, 2016). Estas estructuras no convienen a la lógica y dinámica del capital global, dado que, en 
la medida en que dichas prácticas se encuentren descolonizadas, serán "disfuncionales para la continuación de la globalización capitalista y contrarias a su transformación en "capital humano" económicamente aprovechable" (Liebel, 2016, p. 263).

Liebel (2016) ilustró el aprovechamiento que se da al ideario de infancia nacida con la sociedad burguesa europea, sirviendo como herramienta para desvalorizar e invisibilizar formas distintas de infancia e incluso erradicarlas mediante políticas púbicas y las ONG, en un ejercicio de racionalidad instrumental y conceptual/teórica. La presión ejercida por instituciones como la Organización Internacional del Trabajo sobre gobiernos locales termina criminalizando el trabajo infantil, bajo el paraguas de los derechos del niño y la protección; se sumerge a la infancia trabajadora en un margen de ilegalidad, propia de la racionalidad tutelar. Esta visión poscolonialista se puede encuadrar dentro de la racionalidad crítica y los postulados postgarantistas.

En esta línea, Morales y Magistris (2019) recuperan una perspectiva co-protagonista de una infancia no hegemónica, aquella capaz de actuar sociopolíticamente desde los márgenes y construir ciudadanías no oficiales, rebasando lo otorgado (insuficiente) por la Convención Internacional sobre los Derechos del Niño (CIDN) y conquistando derechos por ello(as) mismo(as) (postgarantismo). Coto (2012) criticó los modelos ideológicos que legitiman y defienden una condición "pre-ciudadana" de la infancia, mediante la cual se restringe la participación políticosocial infantil. De dicho análisis surge la necesidad de marcos normativos alternativos que puedan incentivar el replanteamiento de las relaciones entre el mundo adulto y la infancia (Coto, 2012), con el fin de estimular la participación infantil desde postulados contrahegémonicos, sin desconocer sus obstáculos ${ }^{16}$.

La participación infantil, tradicionalmente, ha sido simplemente de carácter consultivo, dado la creencia de su marcado egocentrismo, creencia derivada de la psicología del desarrollo. En este sentido, para ampliar la participación hacia una ciudadanía infantil, se han propuesto iniciativas como el Principio de Autonomía Progresiva o de Evolución de Capacidades. Este principio postula el deber de los adultos para ofrecer acompañamiento en el aprendizaje de habilidades que le permitirán al infante ejercer, exigir y participar en el plano ciudadano de manera

\footnotetext{
${ }^{16}$ Algunos de los más visibles son la desnaturalización de la protección que se convierte en autoritarismo (por la dependencia de satisfacción de necesidades) y la imagen id́lica del infante como ser vulnerable, inocente y puro, derivado de la tradición judeocristiana, disminuyendo u ocultando otros atributos como su fuerza, sus conocimientos y su capacidad de decisión (Coto, 2012).
} 
progresiva. La propuesta de Coto (2012) para la ampliación de la participación infantil está atada y depende directamente de la voluntad o, al menos, la permisividad adulta, ligada también al corte garantista y a la racionalidad sustantiva, en tanto que reconoce el valor ciudadano y político del infante.

La teorización y conceptualización de la ampliación de la participación infantil es un reto considerable. Osorio (2016) presentó un bosquejo teórico con el fin de respaldarla en el caso de México, con base en postulados garantistas y rescatando la tipología de Trilla y Novela, basada en Hart (1993), donde se clasifican cuatro tipos de participación infantil, a saber: simple, consultiva, proyectiva y metaparticipación. La participación y el ejercicio efectivo de sus derechos se refuerzan desde la democracia y, al mismo tiempo, dicho ejercicio de derechos refuerza la democracia en sí misma, en una relación recursiva. Con ello, se presenta la posibilidad de que el conocimiento y el ejercicio de derechos, por parte de niños y niñas, generen espacios para criticar normas de la vida social en general, así como las que ellos mismos consideren injustas para ciertos grupos o personas.

Además de los anteriores, se pueden encontrar ya variados estudios, sobre todo recientes, encuadrados dentro la corriente contrahegemónica y la racionalidad crítica. Entre ellos, es posible hallar enfoques que abordan la necesidad de resignificación de las luchas y los lenguajes asociados a la infancia (Bustelo, 2007), al infante como sujeto subalterno y complejo (Vergara et al., 2015); como sujeto de derechos ampliados -incluidos los derechos al territorio, al espacio urbano y natural (Ceballos, 2018); la transformación de la infancia (Gauchet, 2004, Gavarini, 2011); el poder de acción propio en la niñez (Latour, 2006); la individualización desde la experiencia (Prout, 2008); la redefinición de las fronteras y el estatus de la infancia en la modernidad (Renaut, 2004); la cultura de la infancia (Delalande, 2009); entre otros que, concentran su objeto de estudio en la infancia con una mirada renovada, cuestionando lo tutelar.

Algunos autores como Duarte (2016) habían evidenciado ya cierto influjo bidireccional entre instituciones latinoamericanas (dimensión material) y lo cultural (dimensión simbólica). Demostrando que lo simbólico otorga, a través de la cultura, legitimación al sistema de dominación intergeneracional, mientras que en la segunda dimensión se evidencian despliegues de procesos económicos y políticos institucionales, donde se definen quiénes y cómo se toman decisiones, quiénes y cómo se producen, delimitando accesos y clausuras a determinados bienes basados en clases de edades (Duarte, 2016). Además, autoras y autores como González (2015), Fernández 
(2014), Martínez y Muñoz (2015) concentraron sus investigaciones sobre las racionalidades imperantes en las políticas y procesos socializadores de la infancia en Uruguay, Argentina y Chile, encontrando también relaciones relevantes entre discursos científico-académicos y discursos político-institucionales.

En ese sentido, el presente trabajo encuentra hallazgos similares a los anteriores; sin embargo, difiere de los mencionados, en tanto que: 1) incorpora en su abordaje las "mutaciones" de las racionalidades, lo que permite hallar en el surgimiento de lo crítico un proceso influido por irrupciones intelectuales y sociales contrahegemónicas; 2) esboza las posibilidades de una contraposición crítica frente a las racionalidades de corte adultocentrista; y 3) propone englobar y diferenciar las inclinaciones racionales en dos grandes bloques analíticos-conceptuales, la racionalidad tutelar y la racionalidad crítica.

Es precisamente en el punto número tres donde descansan las posibilidades prácticas de este estudio, en tanto que dichos bloques analíticos-conceptuales pueden constituirse como categorías viables para la realización de análisis concernientes al tratamiento de la infancia en distintos escenarios sociales, como en investigaciones académicas futuras, pero también como referencia para la reformulación de políticas dirigidas a la infancia y sus contextos socializadores. En este sentido, por su naturaleza documental, la investigación tiene objetivos e implicaciones teórico-conceptuales que contribuyen al debate sobre la interpretación de la niñez en el contexto actual.

\section{Conclusiones}

La concatenación de racionalidades, categorizada desde los presupuestos weberianos, nutre el gran bloque analítico-conceptual identificado aquí como racionalidad tutelar. A través de ella se experimenta una racionalización del tutelaje, siendo ello determinante en los procesos de adopción y reproducción institucional de políticas y comportamientos adultocentristas, dando cuenta de un influjo bidireccional entre ciencias e instituciones. La hegemonía de la racionalidad tutelar durante el siglo XX en Europa y América ha complejizado y dificultado una comprensión ampliada de la infancia, entorpeciendo o minimizando otros tipos de abordajes distintos al hegemónico. La racionalidad tutelar, central en el influjo mutuo ciencias-instituciones, ha 
necesitado constantes actualizaciones y revalidaciones. En dichos procesos, la actuación del Estado ha sido fundamental, actuación no exenta de disputas y contradicciones particulares en cada sociedad, pero que, sin embargo, terminaron por instalar a la infancia en un terreno que sirvió para reproducir estereotipos de orden, lenguaje, acción, útiles para los poderes en turno.

A partir de la década de los ochenta del siglo XX surgen y toman fuerza posturas, como el interaccionismo, punto de quiebre hacia la mutación de la racionalidad desde las ciencias. Así, la infancia va desprendiéndose metodológicamente de sus espacios socializadores, hasta llegar a ser considerada en sí misma como una construcción social, llegando a cuestionar incluso el insuficiente paradigma garantista, e intentando ser trascendida a través de distintas proposiciones contrahegemónicas recientes, enmarcadas dentro de una racionalidad crítica que camina hacia un horizonte postgarantista. Sin embargo, la aparición de la racionalidad crítica en el escenario académico no ha conllevado aún a la consolidación de dicha racionalidad como una opción epistemológica firme. Ello responde, entre otros, a la enorme dificultad que representa desmontar -acaso cuestionar- el sistema de valores tutelares tan profundamente arraigados, científica y socialmente, valores derivados de un proceso histórico en occidente.

En este sentido, la consolidación de la infancia como grupo determinante y participativo socialmente desde la reflexividad y la organización propia, parece estar encaminada a irrumpir desde escenarios que van más allá de los marcos regulatorios tradicionales y tutelares; es decir, desde proyectos contrahegemónicos de resistencias, de memorias, de decolonizaciones, de reexistencias, de contranarraciones, que desborden la normalidad sociopolítica basada en la racionalidad tutelar. La agencia, como concepto proveniente y profundamente incrustado en variadas teorías sociológicas, parece representar un punto de inflexión importante para las nuevas proposiciones de la racionalidad crítica en los estudios de la infancia.

\section{Referencias}

Alvarado, S., Gómez, A., Ospina, M., y Ospina, H. (2014). La hermenéutica ontológica política o hermenéutica performativa: una propuesta epistémica y metodológica. Revista Nómadas, (40), 206-219. https://www.redalyc.org/pdf/1051/105131005014.pdf 
Aversa, M. (2016). Las tramas sociales de la minoridad: infancias pobres y oficios "deshonestos" en la ciudad de Buenos Aires, fines del siglo XIX y principios del XX. Trashumante.

Revista Americana de Historia 132-153. https://www.redalyc.org/articulo.oa?id=455646948008

Bajoit, G. (2008). La renovación de la sociología contemporánea. Revista Cultura y Representaciones Sociales, 3(5), 9-31. http://www.scielo.org.mx/pdf/crs/v3n5/v3n5a1.pdf

Ballatán, G., y Campanini, S. (2008). La participación política de niños y jóvenes adolescentes: Contribución al debate de la democratización de la escuela. Cuadernos de Antropología Social, (28), 85-106. https://www.redalyc.org/pdf/1809/180913915005.pdf

Bell, D. (1973). The Coming of Post-Industrial Society: A Venture in Social Forecasting. Basic Books.

Bourdieu, P., y Passeron, J. (2008). La Reproducción. Elementos para una teoría del sistema de enseñanza. Editorial Popular.

Bustelo, E. (2007). El recreo de los niños. Argumentos para otro comienzo. Siglo XXI.

Cannella, G., y Viruru, R. (2004). Childhood and Postcolonization: Power, Education, and Contemporary Practice. Routledge Falmer.

Capera, J., y Ñañez, J. (2017). Una mirada crítica al concepto de dominación y racionalidad en el pensamiento de Max Weber. Revista Faia, 6(27), 1-14. https://dialnet.unirioja.es/servlet/articulo?codigo $=5845471$

Ceballos, L. (2018). La infancia y el hábitat urbano informal. Reflexiones sobre Bogotá, Colombia. Bitácora, 28(1),

85-92. https://revistas.unal.edu.co/index.php/bitacora/article/view/57890/pdf

Coto, M. (2012). La agencia de la niña y el niño en la condición pre-ciudadana. Revista Actualidades Investigativas en Educación, 12(2), 1-19. https://www.redalyc.org/pdf/447/44723437019.pdf

Delalande, J. (2009). Les enfants entre eux: Des jeux, des règles, une morale. Autrement.

Duarte, C. (2016). Genealogía del adultocentrismo. La constitución de un patriarcado adultocéntrico. En C. Duarte, y C. Álvarez (Eds.), Juventudes en Chile. Miradas de jóvenes que investigan (pp. 17-47). Social ediciones. 
Dubaniewicz, A. (1997). Abandono de menores. Historia y problemática de las instituciones de protección. edición de la autora.

Durkheim, E. (1956). Education and sociology. Macmillan. (obra original publicada en 1922).

Escobar, H. (2020). La racionalidad instrumental como génesis de la conciencia tecnocrática. Revista Lumen Gentium, 2(2), 52-66. https://revistas.unicatolica.edu.co/revista/index.php/LumGent/article/view/153/90

Fávero, S. M. (2018). Sobre padres e hijos en la ley brasileña: una mirada histórica (1916-2009). En L. Lionetti, I. Cosse y M. Zapiola (Comps.), La historia de las infancias en América Latina (pp. 225-239). Tandil.

Fernández, S. (2014). Integralidades en juego. Racionalidades y tecnologías de gobierno de la infancia en la promoción y protección de derechos de niñas, niños y adolescentes. Revista Pilquen, 17(2), 1-16. https://www.redalyc.org/pdf/3475/347532483008.pdf

Fonseca, J., y Prieto, J. (2020). Marx y Weber: El ethos de la burguesía y los orígenes del capitalismo moderno. Revista Desafios, 32(2), 1-28. https://doi.org/10.12804/revistas.urosario.edu.co/desafios/a.8183

Gaitán, L. (2006a). La nueva sociología de la infancia. Política y Sociedad, 43(1), 9-26. https://revistas.ucm.es/index.php/POSO/article/view/POSO0606130009A/22625

Gaitán, L. (2006b). Sociología de la Infancia: nuevas perspectivas. Síntesis.

Gaitán, L. (Julio de 2013). Socialización e infancia en la teoría sociológica. Trabajo presentado en XI Congreso Español de Sociología, Madrid. https://www.academia.edu/37460283/SOCIALIZACI\%C3\%93N_E_INFANCIA_EN_LA _TEOR\%C3\%8DA_SOCIOL\%C3\%93GICA_1

García, P. (2010). Niñez indígena. Derechos y trabajo infantil. En Organización Internacional del Trabajo (Ed.), Niñez Indígena en América Latina. Situación y Perspectivas (pp. 17-108). http://white.lim.ilo.org/ipec/documentos/publi_encuentro_final.pdf

Gauchet, M. (2004). L’enfant du désir. Le débat, 1(132), 98-121. http://ledebat.gallimard.fr/articles/2004-5-1-enfant-du-desir/

Gavarini, L. (2011). L'enfant, symptôme de la difficulté d'être parent aujourd'hui? En D. Coum (Ed.), Que veut dire être parent aujourd'hui? (pp. 95-108). Érès. 
Gee, J. P. (2005). La ideología en los discursos, lingüística social y alfabetizaciones (2a. ed., P. Manzano, Trad.). Fundación Paideia Galiza.

Giddens, A. (1995). La Constitución Social. Bases para la teoría de la estructuración. Amorrortu. (obra original publicada en 1984).

González, D. (2015). El gobierno de la niñez y la adolescencia en situación de calle: un estudio de la racionalidad de las políticas sociales focalizadas (tesis de maestría). Universidad de la República.

Gramsci, A. (1999). Cuadernos de la cárcel. Era.

Gstettner, P. (1981). The conquest of the child by science. From the history of discipline. Rowohlt.

Jenks, C. (1982). The Sociology of childhood: essential readings. Batsford Academic and Educational.

Jenks C. (1989). Social Theorizing and the Child: Constraints and Possibilities. En S. Doxiadis., \& S. Stewart (Eds.), Early Influences Shaping The Individual (pp. 93-102). NATO ASI Series (Series A: Life Sciences).

Jenks, C. (2005). Childhood (2 Ed.). Routledge.

Jenks, C. (2009). Constructing childhood sociologically. En M. Kehily (Ed), An introduction to Childhood Studies (2 ed.). McGraw-Hill. https://doi.org/10.1080/09540250903192432

Kuhn, T. (1982). La tensión esencial: Estudios selectos sobre la tradición y el cambio en el ámbito de la ciencia. Fondo de Cultura Económica.

Latour, B. (2006). Changer de société, refaire de la sociologie. La Découverte.

Liebel, M. (2016). ¿Niños sin Niñez? Contra la conquista poscolonial de las infancias del Sur global. Millcayac Revista Digital de Ciencias Sociales, 3(5), 245-272. http://revistas.uncu.edu.ar/ojs/index.php/millca-digital/article/view/770

Lionetti, L. (2018). La construcción del campo de la infancia anormal en Argentina. Discursos, representaciones y prácticas profesionales. En L. Lionetti, I. Cosse y M. Zapiola (Comps.), xx La historia de las infancias en América Latina (pp. 47-72). Tandil.

Llobet, V. (2010). ¿Fábricas de niños? Las instituciones en la era de los derechos. Centro de Publicaciones Educativas y Material Didáctico.

Lozano, M., Mendoza-Toraya, M., Rocha, F., \& Welter, Z. (2016). La Apropiación Social de la Ciencia, la Tecnología y la Innovación (ASCTI): políticas y prácticas en Chile, Colombia, 
Ecuador y Perú. Trilogía Ciencia Tecnología Sociedad, 8(15), 25-40. https://doi.org/10.22430/21457778.406

Mannheim, K. (2012). Le Problème des générations. Éditions Armand Colin. (Obra original publicada en 1928).

Martínez, M., y Muñoz, G. (2015). Construcción de imaginarios de la infancia y formación de educadoras de párvulos. Revista Latinoamericana de Ciencias Sociales, Niñez y Juventud, 13(1), 343-355. $\quad$ http://www.scielo.org.co/scielo.php?pid=S1692 $\underline{715 X 2015000100021 \& \text { script }=\text { sci abstract\&tlng=es }}$

Mead, G. H. (1982). Espíritu, persona y sociedad: desde el punto de vista del conductivismo social. PaidÓs.

Mendel, G. (1971). Pour décoloniser l'enfant. Sociopsychanalyse de l'autorité. Payot.

Morales, S., y Magistris, G. (2019). El co-protagonismo como nuevo paradigma de infancia: Hacia un horizonte emancipatorio en las relaciones intergeneracionales. Kairos, 23(44), 1-21. http://www.revistakairos.org/wp-content/uploads/morales-magistris.pdf

Neustadter, R (1989). Phone Home: From Childhood Amnesia to the Catcher in Sci-Fi-the Transformation of Childhood in Contemporary Science Fiction Films. Youth \& Society, 20(3), 227-240. https://doi.org/10.1177/0044118X89020003001

Osorio, A. (enero-abril, 2016). La ampliación de la participación infantil en México. Una aproximación sociológica a sus razones, obstáculos y condiciones. Sociológica, 31(87), 111-142. http://www.scielo.org.mx/pdf/soc/v31n87/v31n87a4.pdf

Parsons, T. (1951). The social system. Routledge.

Pavez, I., y Sepúlveda, N. (agosto, 2019). Concepto de agencia en los estudios de infancia. Una revisión teórica. Sociedad e Infancias, 3(1), 193-210. https://doi.org/10.5209/soci.63243

Piaget, J. (1972a). La psicología de la inteligencia. Crítica.

Piaget, J. (1972b). Psicología y epistemología. Emecé editores.

Plesnicar, L. N. (julio-diciembre, 2017). Las infancias en el orden global desigual. Diálogo con Manfred Liebel (entrevista). Revista Latinoamericana de Ciencias Sociales, Niñez y Juventud, 15(2), 1332-1336. https://www.redalyc.org/pdf/773/77352074038.pdf 
Polisena, M. (2018). La racionalización en Occidente: experiencia del desencanto del mundo: Max Weber y George Bataille. Eikasia Revista de Filosofía, (86), 131-139. http://www.revistadefilosofia.org/84-06.pdf

Prout, A. (2008). Culture-nature and the construction of childhood. En K. Drotner, y S. Livingstone (Eds.), The international handbook of children, media and culture (pp. 21-35). Sage.

Punch, S. (2001). Negotiating Autonomy: Childhoods in Rural Bolivia. En L. Alanen, y B. Myall (Eds.), Conceptualising Child-Adult Relations (pp. 23-36). Routledge Falmer.

Pussetto, M. (2016). Entre niñez, estado y adultocentrismo. Cercanías y distancias desde una práctica extensionista. Crítica y Resistencias, (2), 188-205. https://www.criticayresistencias.com.ar/revista/article/view/78/75

Renaut, A. (2004). La fin de l'autorité. Flammarion.

Rodríguez, I. (2003). Condicionantes teóricos en el surgimiento de la sociología de la infancia: el caso la la sociología Española. http://www.derechosinfancia.org.mx/Temas/temas_sociologia1.htm

Rodríguez, I. (2007). Para una sociología de la infancia: aspectos teóricos y metodológicos. Centro de Investigaciones Sociológicas.

Sayago, S. (2014). El análisis del discurso como técnica de investigación cualitativa y cuantitativa en las ciencias sociales. Cinta de Moebio, (49), 1-10. https://www.redalyc.org/articulo.oa?id=10131417001

Strauss, A., y Corbin, J. (2002). Bases de la investigación cualitativa. Técnicas y procedimientos para desarrollar la teoría fundamentada. Universidad de Antioquia.

Vergara, A., Peña, M., Chávez, P., y Vergara, E. (2015). Los niños como sujetos sociales: El aporte de los Nuevos Estudios Sociales de la infancia y el Análisis Crítico del Discurso. Psicoperspectivas, $14(1)$, $55-65$. https://www.psicoperspectivas.cl/index.php/psicoperspectivas/article/viewFile/544/408

Villegas, F. G. (2000). El concepto de racionalidad en Max Weber. En C. Trueba (Coord.), Racionalidad, lenguaje, argumento y acción (pp.229-242). UAM.

Weber, M. (1983). Ensayos sobre sociología de la religión. Taurus.

Weber, M. (1992). La ética protestante y el espíritu del capitalismo. Península. 
Wetherell, M., y Potter, J. (1996). El análisis de discurso y la identificación de reportorios interpretativos. En A.J. Gordo y J. Linaza (Coords.), Psicologías, Discursos y Poder (pp.6378). Visor.

Williams, R. (1997). Marxismo y literatura. Península.

Wrong, D. (1961). The Oversocialized Conception of Man in Modern Sociology. American Sociological Review, 26(2), 183-193. http://www.jstor.org/stable/2089854

Zapiola, M. C. (2018). Estado e infancia en Argentina: reflexiones sobre un recorrido historiográfico. En L. Lionetti, I. Cosse y M. Zapiola (Comps.), xx La historia de las infancias en América Latina (pp. 91-112). Tandil. 